\title{
Wave stability in isotropic temperature-rate-dependent thermoelasticity*
}

\author{
Amnah M. Alharbi ${ }^{\dagger}$ and Nigel H. Scott
}

April 6, 2016

\begin{abstract}
Temperature-rate-dependent thermoelasticity is a theory of thermoelasticity in which the stress, entropy and heat flux are permitted to depend on the rate of change of temperature and the temperature gradient as well as the usual variables temperature and deformation gradient. This has the effect of introducing two relaxation times into the equations of thermoelasticity. Another important effect is that heat now travels at a finite speed rather than the infinite speed implied by the diffusion equation. In an isotropic temperature-rate-dependent thermoelastic material it is found that four plane harmonic waves may propagate, two purely elastic transverse waves and two longitudinal waves which are dispersive and attenuated. All four waves are stable in the sense that their amplitude remains bounded. An alternative theory that forces heat to travel at finite speed is generalized thermoelasticity in which the rate of change of heat flux also appears in the heat conduction equation, thereby introducing a relaxation time. Two different methods of combining the effects of temperature-rate-dependent thermoelasticity and generalized thermoelasticity are discussed and it is found that the transverse waves are unaltered but that one or both of the longitudinal waves become unstable.
\end{abstract}

\section{Keywords}

Thermoelasticity, generalized thermoelasticity, second sound, harmonic waves, stability, two temperatures, two relaxation times.

\section{Introduction}

The theory of generalized thermoelasticity emerged over the last few decades to overcome the drawback of classical thermoelasticity which predicts infinite speeds for thermal disturbances. This is regarded as unrealistic from a physical point of view.

At present there are two different models of generalized thermoelasticity which are being extensively used, both being modifications of the classical theory of thermoelasticity. The first, proposed by Lord and Shulman [1], involves a modification of Fourier's heat

\footnotetext{
*To appear in: Mathematics and Mechanics of Solids, (2016), DOI: 10.1177/1081286516638778

$\dagger$ Department of Mathematics, Faculty of Science, Taif University, Saudi Arabia. Email: alharbi.a.m@hotmail.com

${ }^{\ddagger}$ Corresponding author: School of Mathematics, University of East Anglia, Norwich Research Park, Norwich NR4 7TJ, UK. Email: n.scott@uea.ac.uk
} 
conduction equation by incorporating into that equation a term dependent on the rate of change of heat flux. This introduces a relaxation time into the equations of thermoelasticity. We refer to the Lord and Shulman [1] theory as generalized thermoelasticity. The second model, proposed by Green and Lindsay [2], allows the stress, entropy and heat flux to depend on the rate of change of temperature and the temperature gradient, in addition to the temperature and deformation gradient. This has the effect of introducing two relaxation times into the equations of thermoelasticity. A noteworthy feature of the Green and Lindsay [2] theory is that it preserves the classical Fourier equation of heat conduction provided that the material has a centre of symmetry at each point. Furthermore, it implies the symmetry of the heat conduction tensor. We refer to the Green and Lindsay [2] theory as temperature-rate-dependent thermoelasticity.

In both of the above theories the hyperbolic-parabolic equations of classical thermoelasticity are replaced by purely hyperbolic systems of equations in which all disturbances, including thermal ones, propagate at finite speed. This extra wave propagating at finite speed is known as second sound.

In his book on heat waves Straughan [3] discusses at length the theories of Lord and Shulman [1] and Green and Lindsay [2], together with other theories involving the finite-speed propagation of heat effects. The development of the second sound effect has been reviewed also by Chandrasekharaiah [4].

Chandrasekharaiah and Keshavan [5] have introduced a certain linear combination of the equations of classical thermoelasticity and temperature-rate-dependent thermoelasticity in order to provide a unified system of governing equations which we shall explore as a possible model for generalized temperature-rate-dependent thermoelasticity. Ignaczak [6], on the other hand, provides a model for isotropic generalized temperaturerate-dependent thermoelasticity which is a rationally based combination of generalized thermoelasticity and temperature-rate-dependent thermoelasticity in the isotropic case. We generalize his results to the anisotropic case.

A small-amplitude wave form is said to be linearly stable if it remains of uniformly bounded amplitude in the direction of propagation. In any material we might expect an initial small disturbance either to decay to zero or remain bounded as time increases. Thus we might regard the linear stability of wave forms as being a possible criterion for physically reasonable response. Leslie and Scott have demonstrated the linear stability of isotropic classical thermoelasticity [7, section 2] and also of isotropic generalized thermoelasticity [8, section 2]. The former topic was discussed also by Chadwick [9].

The present work is a study of the wave stability of isotropic materials in the context of temperature-rate-dependent thermelasticity and the two generalized thermoelasticity theories that are mentioned above. In Section 2 we derive the secular equation for plane harmonic waves in the temperature-rate-dependent thermoelasticity theory of Green and Lindsay [2]. We find that there are two longitudinal waves and that both are stable in the sense that they propagate with bounded amplitude. Similar calculations are performed in Section 3 for Chandrasekharaiah and Keshavan's [5] unified system of governing equations, considered here as a possible model of generalized temperaturerate-dependent thermoelasticity, but we now find that one longitudinal wave is stable and the other unstable. Similarly, in Section 4 for Ignaczak's [6] model of generalized temperature-rate-dependent thermoelasticity we find that either one longitudinal wave is stable and the other unstable or that both longitudinal waves are unstable. The final section contains a discussion of our results. 


\section{Temperature-rate-dependent thermoelasticity}

The system of four field equations of linear classical thermoelasticity for a homogeneous and anisotropic material is, see Chadwick [10],

$$
\begin{aligned}
& \tilde{c}_{i j k l} u_{k, j l}-\beta_{i j} \theta_{, j}=\rho \ddot{u}_{i}, \\
& k_{i j} \theta_{, i j}-T \beta_{i j} \dot{u}_{i, j}=\rho c \dot{\theta},
\end{aligned}
$$

in which $\tilde{c}_{i j k l}$ are the isothermal elasticity tensor components, $\beta_{i j}$ are the components of the temperature coefficient of stress, $k_{i j}$ are the components of the thermal conductivity tensor, and $c$ is the specific heat at constant deformation. The displacement components $u_{k}$ and the temperature increment $\theta$ depend on position $\mathbf{x}$ and time $t$. The constants $\rho$ and $T$ denote density and absolute temperature in the equilibrium state, respectively. The superposed dot is a time partial derivative and ()$_{, j}$ denotes the space partial derivative $\partial() / \partial x_{j}$ and the summation convention is applied over twice-occurring subscripts.

The system of field equations of linear temperature-rate-dependent thermoelasticity for a homogeneous and anisotropic material is

$$
\begin{gathered}
\tilde{c}_{i j k l} u_{k, j l}-\beta_{i j}\left(1+\alpha_{1}^{\prime} \frac{\partial}{\partial t}\right) \theta_{, j}=\rho \ddot{u}_{i}, \\
k_{i j} \theta_{, i j}-T \beta_{i j} \dot{u}_{i, j}=\rho c\left(1+\alpha_{0}^{\prime} \frac{\partial}{\partial t}\right) \dot{\theta},
\end{gathered}
$$

see Green and Lindsay [2] or Straughan [3]. The material constants $\alpha_{1}^{\prime}, \alpha_{0}^{\prime}$ are relaxation times satisfying

$$
\alpha_{1}^{\prime} \geq \alpha_{0}^{\prime} \geq 0
$$

these inequalities coming from the second law of thermodynamics, see [2]. Taking $\alpha_{1}^{\prime}=$ $\alpha_{0}^{\prime}=0$ reduces $(2)$ to $(1)$.

For an isotropic thermoelastic body the components $\tilde{c}_{i j k l}, \beta_{i j}$ and $k_{i j}$ take the simple forms

$$
\tilde{c}_{i j k l}=\tilde{\lambda}^{\prime} \delta_{i j} \delta_{k l}+\tilde{\mu}^{\prime}\left(\delta_{i k} \delta_{j l}+\delta_{i l} \delta_{j k}\right), \quad \beta_{i j}=\beta \delta_{i j}, \quad k_{i j}=k \delta_{i j},
$$

in which $\tilde{\lambda}^{\prime}$ and $\tilde{\mu}^{\prime}$ are the isothermal Lamé elastic moduli, $\delta_{i j}$ denote the components of the unit tensor, $\beta$ is the scalar temperature coefficient of stress and $k$ is the scalar thermal conductivity. Inserting (4) into (2) gives the field equations for an isotropic temperature-rate-dependent thermoelastic material:

$$
\begin{aligned}
& \left(\tilde{\lambda}^{\prime}+\tilde{\mu}^{\prime}\right) u_{j, i j}+\tilde{\mu}^{\prime} u_{i, j j}-\beta\left(\theta_{, i}+\alpha_{1}^{\prime} \dot{\theta}_{, i}\right)=\rho \ddot{u}_{i}, \\
& k \theta_{, j j}-T \beta \dot{u}_{j, j}=\rho c\left(\dot{\theta}+\alpha_{0}^{\prime} \ddot{\theta}\right) .
\end{aligned}
$$

If $\beta \neq 0$ the displacements $\mathbf{u}$ and temperature increment $\theta$ are coupled together in these equations. However, if $\beta=0$ the system (5) decouples into (5) ${ }_{1}$, a hyperbolic system of isothermal elastic wave propagation, and $(5)_{2}$, the usual equation of heat conduction with the addition of a relaxation time $\alpha_{0}^{\prime}$.

\subsection{The secular equation}

Now we seek solutions of (5) in the form of harmonic plane waves

$$
\left\{u_{i}, \theta\right\}=\left\{U_{i}, \Theta\right\} \exp \left\{i \omega^{\prime}(s \mathbf{n} \cdot \mathbf{x}-t)\right\}
$$


where $\omega^{\prime}$ is the angular frequency and $\mathbf{n}$ is the unit wave normal vector in the direction of propagation, both of which are real constants. The amplitudes $\left\{U_{i}, \Theta\right\}$ and slowness $s$ are in general complex constants. The slowness $s$ is the reciprocal of the (complex) wave velocity $v: s=1 / v$. If a quantity $Q$ has the $\mathbf{x}$ and $t$ dependence of (6) then derivatives of $Q$ become simply products of $Q$ :

$$
Q, j=\left(i \omega^{\prime} s n_{j}\right) Q, \quad \dot{Q}=\left(-i \omega^{\prime}\right) Q
$$

Inserting (6) into the momentum equation $(5)_{1}$ and dividing by $\gamma\left(i \omega^{\prime} s\right)^{2}$, where $\gamma$ is a positive constant with the physical dimensions of stress, leads, after cancelling the exponential factor, to the three propagation conditions

$$
\left[(\tilde{\lambda}+\tilde{\mu}) n_{i} n_{j}+(\tilde{\mu}-w) \delta_{i j}\right] U_{j}+i \omega^{\prime-1} s^{-1} \gamma^{-1} \beta n_{i}\left(1-i \omega^{\prime} \alpha_{1}^{\prime}\right) \Theta=0,
$$

in which we have defined

$$
\tilde{\lambda}=\frac{\tilde{\lambda}^{\prime}}{\gamma}, \quad \tilde{\mu}=\frac{\tilde{\mu}^{\prime}}{\gamma}, \quad w=\frac{\rho s^{-2}}{\gamma},
$$

the first two of which are dimensionless Lamé moduli and $w$ is effectively a dimensionless squared wave speed.

On inserting the harmonic plane wave forms (6) into the energy equation $(5)_{2}$ and dividing by $\gamma c\left(i \omega^{\prime} s^{2}\right)$ we find, after cancelling the exponential factor, that

$$
\omega^{\prime} s^{-1} \frac{T \beta}{\gamma c} n_{j} U_{j}+\left\{\frac{i \omega^{\prime} k}{\gamma c}+w\left(1-i \omega^{\prime} \alpha_{0}^{\prime}\right)\right\} \Theta=0 .
$$

Eliminating $\Theta$ between equations (7) and (9) leads to

$$
\left\{(\tilde{\mu}-w) \delta_{i j}+\left[\tilde{\lambda}+\tilde{\mu}+\varepsilon \frac{w\left(1-i \omega \alpha_{1}\right)}{w\left(1-i \omega \alpha_{0}\right)+i \omega}\right] n_{i} n_{j}\right\} U_{j}=0,
$$

in which we have introduced the dimensionless quantities

$$
\omega=\frac{\omega^{\prime}}{\omega^{*}}, \quad \alpha_{0}=\alpha_{0}^{\prime} \omega^{*}, \quad \alpha_{1}=\alpha_{1}^{\prime} \omega^{*}, \quad \varepsilon=\frac{T \beta^{2}}{\rho c \gamma},
$$

where $\omega^{*}=\gamma c / k$ has the physical dimensions of frequency. Then $\omega$ is a dimensionless frequency and $\alpha_{0}$ and $\alpha_{1}$ are dimensionless relaxation times such that

$$
\omega^{\prime} \alpha_{0}^{\prime}=\omega \alpha_{0}, \quad \omega^{\prime} \alpha_{1}^{\prime}=\omega \alpha_{1} \quad \text { and } \quad \alpha_{1} \geq \alpha_{0} \geq 0
$$

the inequalities coming from (3). The thermomechanical coupling constant $\varepsilon$ is a dimensionless measure of the degree of coupling between mechanical and thermal effects, so-called because it vanishes with $\beta$.

The requirement that (10) should have non-trivial solutions for $U_{j}, j=1,2,3$, leads to the secular equation

$$
\operatorname{det}\left\{(\tilde{\mu}-w) \mathbf{1}+\left[\tilde{\lambda}+\tilde{\mu}+\varepsilon \frac{w\left(1-i \omega \alpha_{1}\right)}{w\left(1-i \omega \alpha_{0}\right)+i \omega}\right] \mathbf{n} \otimes \mathbf{n}\right\}=0,
$$


where 1 denotes the unit tensor, $\mathbf{n}$ the wave normal vector and $\otimes$ the dyadic product of vectors. We quote from $[11,(\mathrm{~A} 1)]$ the identity

$$
\operatorname{det}(\mathbf{A}+\alpha \mathbf{a} \otimes \mathbf{a})=\operatorname{det} \mathbf{A}+\alpha \mathbf{a} \cdot \mathbf{A}^{\mathrm{adj}} \mathbf{a},
$$

in which $\alpha$, $\mathbf{a}$ and $\mathbf{A}$ are arbitrary quantities and adj denotes the adjugate of a matrix, i.e. the transposed matrix of cofactors. Applying (14) to (13) gives the secular equation in the form

$$
\operatorname{det}\{(\tilde{\mu}-w) \mathbf{1}\}+\left[\tilde{\lambda}+\tilde{\mu}+\varepsilon \frac{w\left(1-i \omega \alpha_{1}\right)}{w\left(1-i \omega \alpha_{0}\right)+i \omega}\right] \mathbf{n} \cdot\{(\tilde{\mu}-w) \mathbf{1}\}^{\text {adj }} \mathbf{n}=0 .
$$

Because

$$
\operatorname{det}\{(\tilde{\mu}-w) \mathbf{1}\}=(\tilde{\mu}-w)^{3} \text { and } \mathbf{n} \cdot\{(\tilde{\mu}-w) \mathbf{1}\}^{\mathrm{adj}} \mathbf{n}=(\tilde{\mu}-w)^{2}
$$

we can rewrite (15) in the form

$$
(\tilde{\mu}-w)^{2}\left[\tilde{\lambda}+2 \tilde{\mu}-w+\varepsilon \frac{w\left(1-i \omega \alpha_{1}\right)}{w\left(1-i \omega \alpha_{0}\right)+i \omega}\right]=0 .
$$

We now select a convenient value for the scaling parameter $\gamma$, namely,

$$
\gamma=\tilde{\lambda}^{\prime}+2 \tilde{\mu}^{\prime}, \quad \text { so that } \quad \tilde{\lambda}+2 \tilde{\mu}=1 \text {, }
$$

and the secular equation (17) becomes in fully non-dimensional form

$$
(w-\tilde{\mu})^{2}\left[1-w+\varepsilon \frac{w\left(1-i \omega \alpha_{1}\right)}{w\left(1-i \omega \alpha_{0}\right)+i \omega}\right]=0 .
$$

The choice $(18)_{1}$ of scaling parameter means that we are comparing all squared wave speeds with the squared wave speed $\left(\tilde{\lambda}^{\prime}+2 \tilde{\mu}^{\prime}\right) / \rho$ of purely elastic longitudinal waves in an isothermal isotropic linearly elastic solid.

The secular equation (19) has the repeated root

$$
w=\tilde{\mu}=\frac{\tilde{\mu}^{\prime}}{\tilde{\lambda}^{\prime}+2 \tilde{\mu}^{\prime}}
$$

which corresponds to two transverse non-dispersive purely elastic isothermal isotropic waves unaffected by heat conduction. Since usually $\tilde{\lambda}^{\prime}>0$ the dimensionless transverse squared wave speed $w=\tilde{\mu}$ is restricted by

$$
0<\tilde{\mu}<\frac{1}{2}
$$

as compared with the dimensionless isothermal longitudinal squared wave speed $w=1$.

After expanding and rearranging the part within square brackets of equation (19) we obtain the following form of the non-dimensional secular equation for longitudinal waves in isotropic temperature-rate-dependent thermoelasticity:

$$
w(w-1-\varepsilon)+i \omega \frac{1+w\left(\alpha_{1}-\alpha_{0}\right)}{1-i \omega \alpha_{1}}(w-1)=0 .
$$


Upon multiplying by $1-i \omega \alpha_{1}$ and collecting terms in $w$ we obtain an alternative form of the secular equation:

$$
w^{2}\left(1-i \omega \alpha_{0}\right)-w\left[1+\varepsilon-i \omega\left(1+\alpha_{0}+\varepsilon \alpha_{1}\right)\right]-i \omega=0 .
$$

This is a quadratic equation for the dimensionless squared wave speed $w$ as a function of the dimensionless frequency $\omega$ and the non-negative dimensionless constants $\alpha_{0}, \alpha_{1}$ and $\varepsilon$. As $\omega$ varies over $0 \leq \omega<\infty$ the two branches of the complex function $w(\omega)$ vary over the complex plane. A wave form is said to be linearly stable if it remains uniformly bounded in the direction of propagation. The condition for linear stability of these wave forms is

$$
\operatorname{Im} w \leq 0
$$

for $0 \leq \omega<\infty$, see $[12,(18)]$.

On taking $\alpha_{1}=\alpha_{0}=0$, (23) reduces to the corresponding secular equation for classical thermoelasticity, see [9, (5)], which is discussed further at $[7,(2.14)]$ and illustrated in Figure 1. It is of interest to note also that on putting $\alpha_{1}=\alpha_{0}$ in (23), and replacing both by $\tau$, there results the secular equation [8, (2.14)] of longitudinal waves in isotropic generalized thermoelasticity. In this sense, then, it can be said that generalized thermoelasticity is a special case of temperature-rate-dependent thermoelasticity. However, the relevant underlying field equations of isotropic temperature-rate-dependent thermoelasticity, namely (5) with $\alpha_{1}^{\prime}=\alpha_{0}^{\prime}$ and both replaced by $\tau_{0}$, are different from those of isotropic generalized thermoelasticity, see [8,(2.3)], even though the secular equations are the same.

The roots of the quadratic equation (23) are given by

$$
w_{0,1}=\frac{z_{1} \mp\left[z_{1}^{2}+4 i \omega\left(1-i \omega \alpha_{0}\right)\right]^{\frac{1}{2}}}{2\left(1-i \omega \alpha_{0}\right)},
$$

where

$$
z_{1}=1+\varepsilon-i \omega\left(1+\alpha_{0}+\varepsilon \alpha_{1}\right) .
$$

If we put $\alpha_{0}=\alpha_{1}=0$ in (25) and (26), we recover the two branches of $w$ which occur in the case of the classical thermoelasticity of an isotropic material, see [7, (2.15)]. Similarly, if we put $\alpha_{0}=\alpha_{1}$ in (25) and (26) and replace both by $\tau$, we recover the two branches of $w$ for isotropic generalized thermoelasticity, see [8, (2.15)].

The uncoupled case $\varepsilon=0$. In this case, the roots of (23) reduce to

$$
w_{0}=\frac{-i \omega}{1-i \omega \alpha_{0}}, \quad w_{1}=1
$$

where $w_{0}$ represents a purely diffusive mode and $w_{1}$ represents an unattenuated, nondispersive longitudinal wave (a purely elastic mode). We can show that as $\omega$ varies over positive values the branch $w_{0}$ describes a semicircle in the lower half $w$-plane if $\alpha_{0}>0$ with centre at $(1 / 2) \alpha_{0}+0 i$ and radius $(1 / 2) \alpha_{0}$, as illustrated in Figure 2(a). 


\subsection{Low frequency expansions}

At zero frequency the roots (25) become, for $\varepsilon>0$,

$$
w_{0}=0, \quad w_{1}=1+\varepsilon,
$$

as is most easily seen by putting $\omega=0$ in (22). The points (28) are marked with a $\times$ on each part of Figures 1-4 and in each case mark the point $\omega=0$ at the beginning of a mode.

Expanding (25) for small $\omega$ we find that

$$
w_{0}=\frac{-i \omega}{1+\varepsilon}+O\left(\omega^{2}\right)
$$

and that

$$
w_{1}=1+\varepsilon-i \omega \varepsilon\left\{\alpha_{1}-\alpha_{0}+\frac{1}{1+\varepsilon}\right\}+O\left(\omega^{2}\right) .
$$

Equation (29) is a diffusive mode and equation (30) is an elastic mode. On putting $\omega=0$ in both these equations we recover (28), as expected. Since $\alpha_{1} \geq \alpha_{0}$ we see that both these branches have negative imaginary part and so are stable at low frequency.

\subsection{High frequency expansions}

Collect terms in $i \omega$ in (23) together:

$$
w(w-1-\varepsilon)-i \omega\left[\alpha_{0} w^{2}-\left(1+\alpha_{0}+\varepsilon \alpha_{1}\right) w+1\right]=0 .
$$

The roots of the secular equation (23) in the high frequency limit, as $\omega \rightarrow \infty$, may be obtained by equating to zero the coefficient of $i \omega$ in (31):

$$
H(w) \equiv \alpha_{0} w^{2}-\left[1+\alpha_{0}+\varepsilon \alpha_{1}\right] w+1=0 .
$$

We now need to determine the positions of the two zeros of $H(w)$ :

$$
\begin{aligned}
& H(0)=1>0, \\
& H(1)=-\varepsilon \alpha_{1}<0, \\
& H(1+\varepsilon)=-\varepsilon-\varepsilon\left(\alpha_{1}-\alpha_{0}\right)-\varepsilon^{2}\left(\alpha_{1}-\alpha_{0}\right)<0, \\
& H(\infty)=\infty>0,
\end{aligned}
$$

so that the equation $H(w)=0$ has two real roots $\bar{h}_{1}$ and $\bar{h}_{2}$ satisfying

$$
0<\bar{h}_{1}<1<1+\varepsilon<\bar{h}_{2}
$$

These are the two real roots of the secular equation (31) in the high frequency limit. They satisfy the quadratic polynomial

$$
\bar{h}(w)=\left(w-\bar{h}_{1}\right)\left(w-\bar{h}_{2}\right),
$$

and we must have

$$
H(w) \equiv \alpha_{0} \bar{h}(w)
$$


The secular equation (31) may now be written as

$$
w(w-1-\varepsilon)-i \omega \alpha_{0}\left(w-\bar{h}_{1}\right)\left(w-\bar{h}_{2}\right)=0 .
$$

For $\omega$ sufficiently large the roots of (34) may be written as

$$
w_{0}=\bar{h}_{1}-i A \omega^{-1}+O\left(\omega^{-2}\right) \quad \text { and } \quad w_{1}=\bar{h}_{2}-i B \omega^{-1}+O\left(\omega^{-2}\right),
$$

where the constants $A$ and $B$ are to be determined. Substituting (35) into (34) gives

$$
A=\frac{\bar{h}_{1}\left(\bar{h}_{1}-1-\varepsilon\right)}{\alpha_{0}\left(\bar{h}_{1}-\bar{h}_{2}\right)}>0 \quad \text { and } \quad B=\frac{\bar{h}_{2}\left(\bar{h}_{2}-1-\varepsilon\right)}{\alpha_{0}\left(\bar{h}_{2}-\bar{h}_{1}\right)}>0,
$$

the fact that $A$ and $B$ are positive coming from the inequalities (33). It follows from the stability condition $\operatorname{Im} w \leq 0$ that both branches are stable in the high frequency limit.

We have shown that both branches are stable at both low and high frequencies and we may further show that both are stable for all frequencies by considering (34) rewritten as

$$
\frac{w(w-1-\varepsilon)}{\alpha_{0}\left(w-\bar{h}_{1}\right)\left(w-\bar{h}_{2}\right)}=i \omega .
$$

For a branch to become unstable at some frequency $\omega$ satisfying $0<\omega<\infty$ would require $w$ to be real at that frequency for then the branch would be passing from $\operatorname{Im} w<$ 0 to $\operatorname{Im} w>0$, see (24). But this is impossible as then (37) would be seeking to equate a real number with a purely imaginary one. Thus both branches are stable for all frequencies.

The high frequency limits $\bar{h}_{1}$ and $\bar{h}_{2}$ are marked with a $\circ$ in Figures $1-2$ except that $\bar{h}_{2}$ is infinite in Figure 1.

\subsection{Intersection of branches}

We see that in most of the plots of Figures 1 and 2 the branches $w_{0}(\omega)$ and $w_{1}(\omega)$ do not intersect for any value of $\omega$, the exceptions being parts (d) of each Figure. These points of intersection correspond to a double root of the secular equation (23) which can occur only when the term $z_{1}^{2}+4 i \omega\left(1-i \omega \alpha_{0}\right)$ in $(25)$ vanishes. The vanishing of the imaginary part of this quantity yields the relation

$$
\alpha_{0}+\varepsilon \alpha_{1}=\frac{1-\varepsilon}{1+\varepsilon}
$$

between the parameters $\alpha_{0}, \alpha_{1}$ and $\varepsilon$, whilst the vanishing of the real part gives the expression

$$
\omega_{c}=\frac{1+\varepsilon}{\sqrt{\left(1+\alpha_{0}+\varepsilon \alpha_{1}\right)^{2}-4 \alpha_{0}}}=\frac{\frac{1}{2}(1+\varepsilon)^{2}}{\sqrt{1-\alpha_{0}(1+\varepsilon)^{2}}}
$$

for the critical frequency $\omega_{c}$ at which the point of intersection occurs, where (38) has been used to eliminate $\varepsilon \alpha_{1}$. For given $\alpha_{0}$ and $\alpha_{1}$, (38) may be regarded as a (quadratic) equation for the critical value $\varepsilon_{c}$ at which the point of intersection occurs.

Since $\alpha_{1} \geq \alpha_{0} \geq 0$ from $(12)_{3},(38)$ shows that

$$
0 \leq \varepsilon_{c} \leq 1
$$


Taking $\varepsilon_{c}=0$ in (38) forces $\alpha_{0}=1$ and $\omega_{c} \rightarrow \infty$ in (39). In fact, if $\varepsilon=0$ there can be no points of intersection as is illustrated in Figures 1(a) and 2(a). If, on the other hand, $\varepsilon_{c}=1$ then (38) forces $\alpha_{1}=\alpha_{0}=0$, the case of isotropic classical thermoelasticity, and the point of intersection occurs at the frequency $\omega_{c}=2$, as illustrated in Figure $1(\mathrm{~d})$. We may therefore conclude that at a point of intersection

$$
0<\varepsilon_{c}<1 \text {. }
$$

Because the right hand side of (38) is less than unity it follows that $\alpha_{0}$ must be selected so that $\alpha_{0}<1$. For any positive choice of $\alpha_{0}$ such that $\alpha_{0}<1$ and any choice of $\alpha_{1}$ such that $\alpha_{1} \geq \alpha_{0}$ it can be shown that (38) can be solved to give a value of $\varepsilon_{c}$ satisfying (40) and that these choices correspond to a point of intersection. This is illustrated in Figure $2(\mathrm{~d})$ in which for the choices $\alpha_{0}=0.1$ and $\alpha_{1}=0.2$ we find that $\varepsilon_{c} \approx 0.6310$ corresponds to a point of intersection.

In the special case $\alpha_{0}=0$, solving (38) for $\varepsilon_{c}$ and employing $(39)_{2}$ gives

$$
\varepsilon_{c}=\frac{2}{\left[\left(1+\alpha_{1}\right)^{2}+4 \alpha_{1}\right]^{1 / 2}+1+\alpha_{1}}, \quad \omega_{c}=\frac{1}{2}\left(1+\varepsilon_{c}\right)^{2}
$$

and this value of $\varepsilon_{c}$ satisfies (40) for any choice of $\alpha_{1}>0$.

\subsection{Numerical results}

In Figures 1 and 2 the roots (25) are plotted as functions of frequency $\omega$ for fixed values of $\varepsilon, \alpha_{0}$ and $\alpha_{1}$. In each subplot of Figures 1 and 2 there is a $\times$ at $w=0$ and $w=1+\varepsilon$, marking the low frequency limits, and a $\circ$ marks the high frequency limits.

In each figure the measure of the degree of thermoelastic coupling, $\varepsilon$, is varied between the subplots for fixed values of $\alpha_{0}$ and $\alpha_{1}$. In all the subplots of Figures 1 and 2 we can see that $\operatorname{Im} w \leq 0$, illustrating the linear stability of each branch of $w(\omega)$ as proved above. Therefore, for isotropic classical thermoelasticity, in which $\alpha_{1}=\alpha_{0}=0$, see Figure 1 , and also for isotropic temperature-rate-dependent thermoelasticity, in which $\alpha_{1} \geq \alpha_{0}>0$, we see that both longitudinal modes are stable. Isotropic classical thermoelasticity is discussed also in [7].

An infinite branch of $w(\omega)$ is one for which $w \rightarrow \infty$ as $\omega \rightarrow \infty$. In Figure 1 we see one finite branch and one infinite branch in each subplot. This occurs because $\alpha_{0}=0$ which leads to $H(w)$ being linear in $w$, rather than quadratic, so that the second high frequency root becomes infinite (i.e. $\bar{h}_{2} \rightarrow \infty$ as $\alpha_{0} \rightarrow 0$ ). We see that for $\varepsilon=0$ the left hand branch lies entire on the negative imaginary axis and so is is diffusive for all frequencies and the right hand branch is elastic for all frequencies. This pattern persists for $\varepsilon>0$ small enough, see parts (b) and (c) of Figure 1. For larger values of $\varepsilon$ the left hand branch is diffusive for low frequencies but elastic for high frequencies and the right hand branch is elastic for low frequencies but diffusive for high frequencies, see parts (e) and (f) of Figure 1. The crossover point is at $\varepsilon=1$ in part $(\mathrm{d})$. This process is described by Chadwick [9].

In Figure 2 both branches are finite. For low frequencies the branch beginning at the origin is diffusive for all values of $\varepsilon$ and both branches are elastic for high frequencies. For $\varepsilon$ small enough the branch beginning at the origin remains diffusive for all frequencies, see parts (a)-(c) of Figure 2. For $\varepsilon$ large enough the branch beginning at the origin becomes elastic at high frequencies but the other branch is elastic at low frequencies and 
becomes diffusive at high frequencies, see parts (e) and (f) of Figure 2. The crossover point is again in part (d) and corresponds to a point of intersection of the two branches as discussed in the previous subsection.

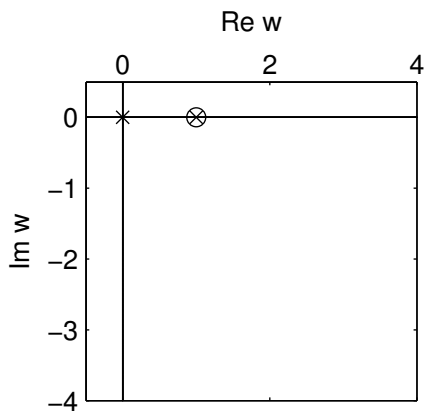

(a) $\varepsilon=0$

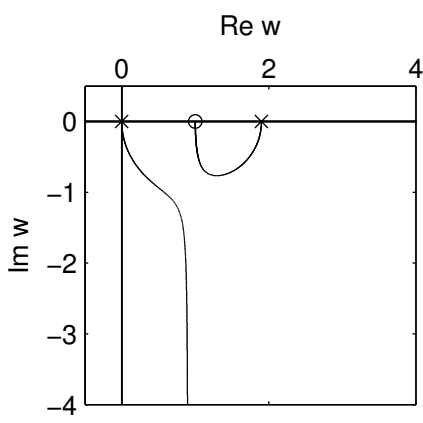

(c) $\varepsilon=0.9$

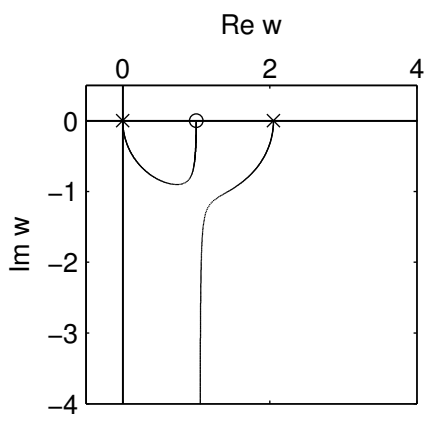

(e) $\varepsilon=1.05$

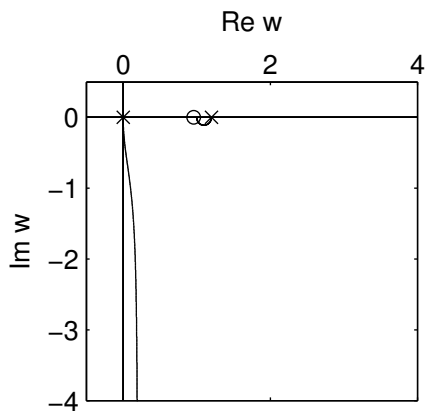

(b) $\varepsilon=0.2$

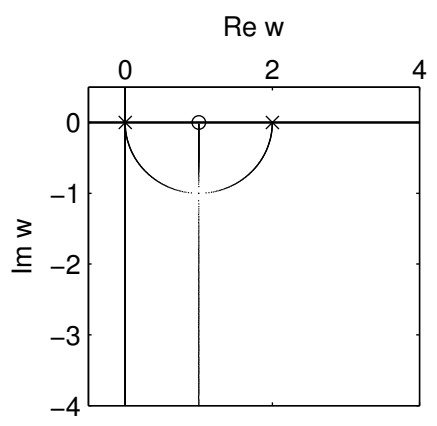

(d) $\varepsilon=1$

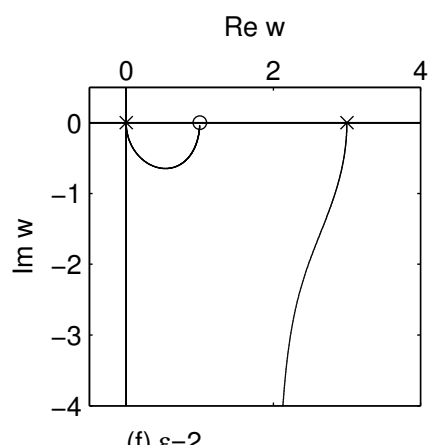

Figure 1: The longitudinal squared wave speeds of isotropic classical thermoelasticity. For each part, $\alpha_{0}=0, \alpha_{1}=0$. 


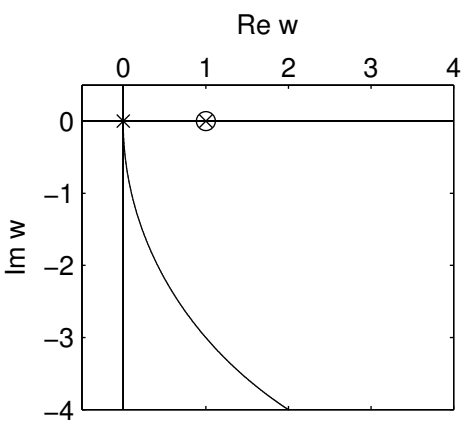

(a) $\varepsilon=0$

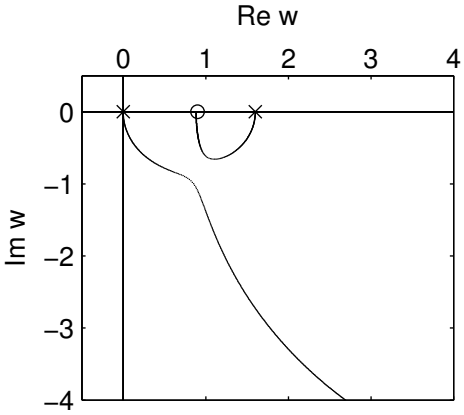

(c) $\varepsilon=0.6$

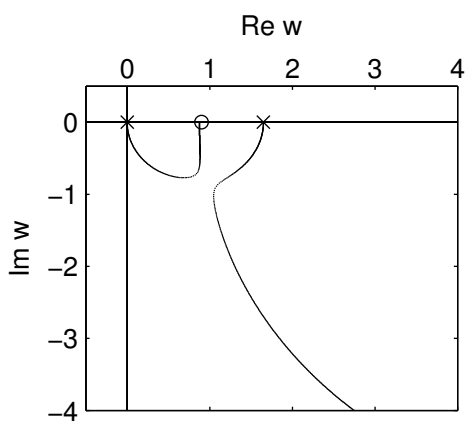

(e) $\varepsilon=0.65$

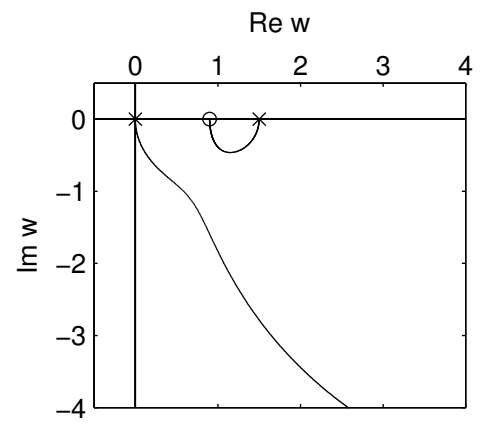

(b) $\varepsilon=0.5$

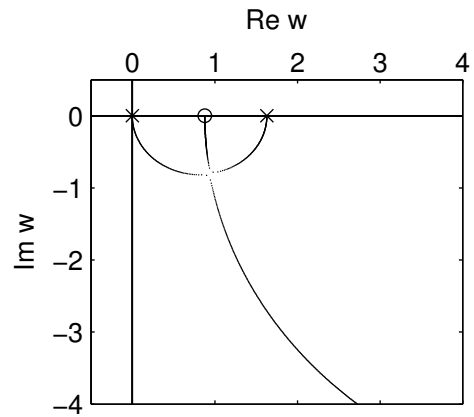

(d) $\varepsilon=0.63$

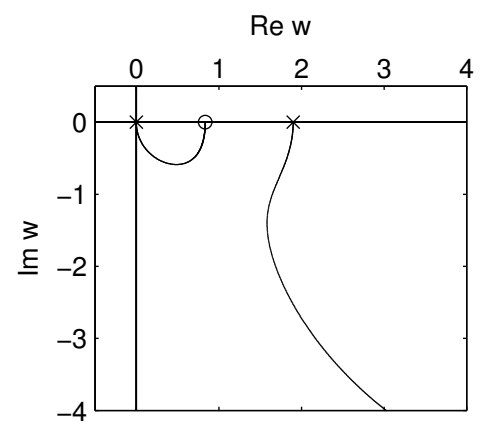

(f) $\varepsilon=0.9$

Figure 2: The longitudinal squared wave speeds of isotropic temperature-rate-dependent thermoelasticity. For each part, $\alpha_{0}=0.1, \alpha_{1}=0.2$. 


\section{Generalized temperature-rate-dependent thermoe- lasticity: Model 1}

For linear generalized thermoelasticity the heat flux vector component $q_{i}$ is given by the constitutive equation

$$
\left(1+\tau_{0}^{\prime} \frac{\partial}{\partial t}\right) q_{i}=-k_{i j} \theta_{, j},
$$

where $\tau_{0}^{\prime}>0$ is a relaxation time. This non-zero $\tau_{0}^{\prime}$ has the effect of leaving $(1)_{1}$ unchanged but replacing $(1)_{2}$ by

$$
k_{i j} \theta_{, i j}=\left(1+\tau_{0}^{\prime} \frac{\partial}{\partial t}\right)\left(T \beta_{i j} \dot{u}_{i, j}+\rho c \dot{\theta}\right),
$$

which is equivalent to

$$
k_{i j} \theta_{, i j}-T \beta_{i j}\left(\dot{u}_{i, j}+\tau_{0}^{\prime} \ddot{u}_{i, j}\right)=\rho c\left(\dot{\theta}+\tau_{0}^{\prime} \ddot{\theta}\right),
$$

see [13]. On taking $\tau_{0}^{\prime}=0$ we see that (42) reduces to the usual Fourier law of heat conduction and that (44) reverts to $(1)_{2}$.

Chandrasekharaiah and Keshavan [5] seek to combine the theories of temperature dependent thermoelasticity and generalized thermoelasticity by considering the following unified system of governing equations

$$
\begin{aligned}
& \tilde{c}_{i j k l} u_{k, j l}-\beta_{i j}\left(\theta_{, j}+\alpha_{1}^{\prime} \dot{\theta}_{, j}\right)=\rho \ddot{u}_{i}, \\
& k_{i j} \theta_{, i j}-T \beta_{i j}\left(\dot{u}_{i, j}+\tau_{0}^{\prime} \ddot{u}_{i, j}\right)=\rho c\left(\dot{\theta}+\alpha_{0}^{\prime} \ddot{\theta}\right),
\end{aligned}
$$

see $[5,(2.3)]$. The first equation is simply $(2)_{1}$ of temperature-rate-dependent thermoelasticity and the second is an ad hoc amalgamation of $(2)_{2}$ and (44) of generalized thermoelasticity in which the second occurrence of $\tau_{0}^{\prime}$ in (44) has been replaced by $\alpha_{0}^{\prime}$. Putting $\tau_{0}^{\prime}=0$ reduces (45) to equation (2) of temperature-rate-dependent thermoelasticity. On the other hand, putting $\alpha_{1}^{\prime}=0$ and $\alpha_{0}^{\prime}=\tau_{0}^{\prime}>0$ gives generalized thermoelasticity but this involves taking $\alpha_{1}^{\prime}<\alpha_{0}^{\prime}$ which contravenes inequality (3) of temperature-rate-dependent thermoelasticity thus exposing the ad hoc nature of the system (45). There is no choice of constitutive assumptions for stress, entropy and heat flux which gives rise to the system (45). We shall refer to this system of equations as generalized temperature-rate-dependent thermoelasticity: Model 1, though (45) was not proposed as such a model by Chandrasekharaiah and Keshavan [5] .

Substituting the isotropic tensor components (4) into (45) gives the field equations of isotropic generalized temperature-rate-dependent thermoelasticity: Model 1.

$$
\begin{aligned}
& \left(\tilde{\lambda}^{\prime}+\tilde{\mu}^{\prime}\right) u_{j, i j}+\tilde{\mu}^{\prime} u_{i, j j}-\beta\left(\theta_{, i}+\alpha_{1}^{\prime} \dot{\theta}_{, i}\right)=\rho \ddot{u}_{i}, \\
& k \theta_{, j j}-T \beta\left(\dot{u}_{i, j}+\tau_{0}^{\prime} \ddot{u}_{i, j}\right)=\rho c\left(\dot{\theta}+\alpha_{0}^{\prime} \ddot{\theta}\right) .
\end{aligned}
$$

\subsection{The secular equation}

Now we look for solutions of (46) of plane harmonic type (6) by inserting (6) into (46) and cancelling the exponential factors. Then divide $(46)_{1}$ by $\gamma\left(i \omega^{\prime} s\right)^{2}$ to get

$$
\left[(\tilde{\lambda}+\tilde{\mu}) n_{i} n_{j}+(\tilde{\mu}-w) \delta_{i j}\right] U_{j}+i \omega^{\prime-1} s^{-1} \beta n_{i}\left(1-i \omega^{\prime} \alpha_{1}^{\prime}\right) \Theta=0
$$


which is exactly the same as (7). From $(46)_{2}$, on dividing by $\gamma c\left(i \omega^{\prime} s^{2}\right)$, we find that

$$
\omega^{\prime} s^{-1} \frac{T \beta}{\gamma c}\left(1-i \omega^{\prime} \tau_{0}^{\prime}\right) n_{j} U_{j}+\left\{\frac{i \omega^{\prime} k}{\gamma c}+w\left(1-i \omega^{\prime} \alpha_{0}^{\prime}\right)\right\} \Theta=0,
$$

which replaces (9). Eliminating $\Theta$ between equations (47) and (48) leads to

$$
\left\{(\tilde{\mu}-w) \delta_{i j}+\left[\tilde{\lambda}+\tilde{\mu}+\varepsilon \frac{w\left(1-i \omega \alpha_{1}\right)\left(1-i \omega \tau_{0}\right)}{w\left(1-i \omega \alpha_{0}\right)+i \omega}\right] n_{i} n_{j}\right\} U_{j}=0,
$$

in which we have introduced the dimensionless quantities (11) as before together with the further dimensionless relaxation time

$$
\tau_{0}=\tau_{0}^{\prime} \omega^{*}, \quad \text { such that } \omega^{\prime} \tau_{0}^{\prime}=\omega \tau_{0} .
$$

Equation (49) can be treated exactly as was (10) in the previous section to obtain the non-dimensional secular equation

$$
(w-\tilde{\mu})^{2}\left[1-w+\varepsilon \frac{w\left(1-i \omega \alpha_{1}\right)\left(1-i \omega \tau_{0}\right)}{w\left(1-i \omega \alpha_{0}\right)+i \omega}\right]=0 .
$$

in place of (19). On putting $\tau_{0}=0$ in (50), we recover (19), as expected. The two transverse waves $w=\tilde{\mu}$ in (50) are as at (20).

After expanding and rearranging the part within square brackets of equation (50) we obtain an alternative form of the non-dimensional secular equation for longitudinal waves in isotropic generalized temperature-rate-dependent thermoelasticity: Model 1.

$$
w(w-1-\varepsilon)+i \omega \frac{1+w\left\{\alpha_{1}-\alpha_{0}+\tau_{0}\left(1-i \omega \alpha_{1}\right)\right\}}{\left(1-i \omega \alpha_{1}\right)\left(1-i \omega \tau_{0}\right)}(w-1)=0 .
$$

This can be rearranged as a quadratic equation in $w$ :

$$
w^{2}\left(1-i \omega \alpha_{0}\right)-w\left[1-i \omega \alpha_{0}+\varepsilon\left(1-i \omega \alpha_{1}\right)\left(1-i \omega \tau_{0}\right)-i \omega\right]-i \omega=0 .
$$

The roots of this quadratic equation are given by

$$
w_{0,1}=\frac{z_{2} \mp\left[z_{2}^{2}+4 i \omega\left(1-i \omega \alpha_{0}\right)\right]^{\frac{1}{2}}}{2\left(1-i \omega \alpha_{0}\right)}
$$

in which

$$
z_{2}=1-i \omega \alpha_{0}+\varepsilon\left(1-i \omega \alpha_{1}\right)\left(1-i \omega \tau_{0}\right)-i \omega
$$

The uncoupled case $\varepsilon=0$. In this case, the roots (53) of (52) reduce to those of the purely temperature-rate-dependent case, namely, equations (27). This explains why Figures 2(a) and 3(a) are the same. 


\subsection{Low frequency expansions}

At zero frequency the roots (53) are again given by (28) for $\varepsilon>0$, as is most easily seen by putting $\omega=0$ in (51). These roots are marked $\times$ in Figure 3 .

From (53) for small $\omega$, we find that

$$
w_{0}=\frac{-i \omega}{1+\varepsilon}+O\left(\omega^{2}\right)
$$

and that

$$
w_{1}=1+\varepsilon-i \omega \varepsilon\left\{\alpha_{1}-\alpha_{0}+\tau_{0}+\frac{1}{1+\varepsilon}\right\}+O\left(\omega^{2}\right) .
$$

Equation (55) represents a diffusive mode and equation (56) an elastic mode. Both modes are stable at low frequency. They should be compared with (29) and (30), respectively, in the temperature-rate-dependent case.

\subsection{High frequency expansions}

The high frequency domain $\omega \rightarrow \infty$ is explored by writing $\zeta=\omega^{-1}$ in (52), multiplying by $\zeta^{2}$ and collecting terms in powers of $\zeta$ to obtain

$$
\zeta^{2} w(w-1-\varepsilon)-i \zeta\left[\alpha_{0} w^{2}-\alpha_{0} w-w \varepsilon\left(\alpha_{1}+\tau_{0}\right)-w+1\right]+w \varepsilon \alpha_{1} \tau_{0}=0 .
$$

The high frequency limit is taken by allowing $\zeta \rightarrow 0$. Putting $\zeta=0$ in (57) reduces it to

$$
w \varepsilon \alpha_{1} \tau_{0}=0
$$

with roots

$$
w_{0}=0, \quad w_{1} \rightarrow \infty,
$$

provided $\varepsilon \alpha_{1} \tau_{0}>0$. Thus in Figure 3 parts (b)-(f) the origin is marked with a $\times$ and a $\circ$ as it is both a low and high frequency limit.

For small $\zeta$ these roots may be better approximated by

$$
w_{0}=i A \zeta+O\left(\zeta^{2}\right), \quad w_{1}=i B \zeta^{-1}+C+O(\zeta)
$$

The constants $A, B$ and $C$ may be obtained by substitution into (57) to arrive finally at the high frequency approximations

$$
w_{0}=\frac{i \omega^{-1}}{\varepsilon \alpha_{1} \tau_{0}}+O\left(\omega^{-2}\right), \quad w_{1}=-\frac{i \omega \varepsilon \alpha_{1} \tau_{0}}{\alpha_{0}}+\frac{1+\alpha_{0}+\varepsilon\left(\alpha_{1}+\tau_{0}\right)-\varepsilon \alpha_{1} \tau_{0} / \alpha_{0}}{2-\alpha_{0}}+O\left(\omega^{-1}\right) .
$$

It is clear that $w_{0}$ is unstable and $w_{1}$ stable in the high frequency limit.

In Figure 3 parts (b)-(f) we see that $w_{1} \rightarrow \infty$ in the lower half $w$-plane as $\omega \rightarrow \infty$, as is indicated by $(60)_{2}$. Also in Figure 3 parts (b)-(f) we see that both branches are stable at low frequency but one is stable and the other unstable at high frequency. Therefore one branch changes from stable to unstable as $\omega$ increases. This is in contrast with Figures 1 and 2 for which all branches are stable. 


\subsection{Intersection of branches}

Points of intersection of the branches of $w(\omega)$ correspond to double roots of the secular equation (52). From its solution (53) we see that this secular equation has a double root if and only if

$$
D \equiv z_{2}^{2}+4 i \omega\left(1-i \omega \alpha_{0}\right)=0
$$

where $z_{2}$ is defined by (54).

Apart from the spurious root $\omega=0$, the vanishing of $\operatorname{Im} D$ gives the following expression for the critical frequency $\omega_{c}$ at which the branches intersect:

$$
\varepsilon \alpha_{1} \tau_{0} \omega_{c}^{2}=1+\varepsilon-\frac{2}{d}
$$

where $d$ is defined by

$$
d=1+\alpha_{0}+\varepsilon\left(\alpha_{1}+\tau_{0}\right) .
$$

The vanishing of $\operatorname{Re} D$ gives the equation

$$
(1+\varepsilon)^{2}-\omega^{2}\left[2(1+\varepsilon) \varepsilon \alpha_{1} \tau_{0}+d^{2}-4 \alpha_{0}\right]+\omega^{4}\left(\varepsilon \alpha_{1} \tau_{0}\right)^{2}=0 .
$$

We now use (61) to eliminate $\omega_{c}^{2}$ from this equation and eventually reduce it to

$$
d[d(1+\varepsilon)-2]\left(d^{2}-4 \alpha_{0}\right)-4 \varepsilon \alpha_{1} \tau_{0}=0
$$

which is quintic in $\varepsilon$ as $d$ is linear in $\varepsilon$. We regard (63) as a quintic equation (with coefficients dependent on the parameters $\left.\alpha_{0}, \alpha_{1}, \tau_{0}\right)$ for the critical value $\varepsilon_{c}$ of $\varepsilon$ for which the branches intersect. We now insert such a root $\varepsilon_{c}$ of (63) into (61) to obtain the critical frequency $\omega_{c}$ at which the branches intersect.

This is illustrated in Figure 3(d) where for the parameter values $\alpha_{0}=0.1, \alpha_{1}=0.2$, $\tau_{0}=0.1$, we find that the critical values giving a point of intersection are $\varepsilon_{c}=0.58$ and $\omega_{c}=2.00$. 


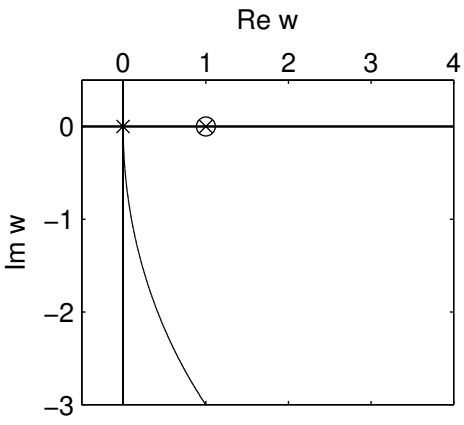

(a) $\varepsilon=0$

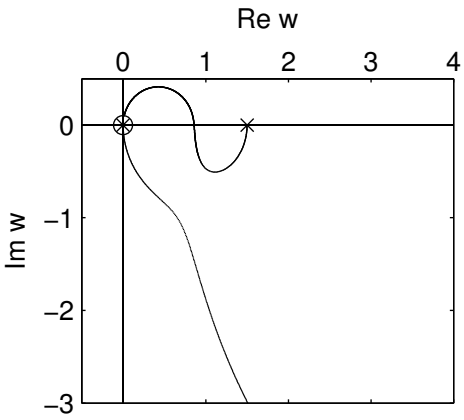

(c) $\varepsilon=0.5$

Re w

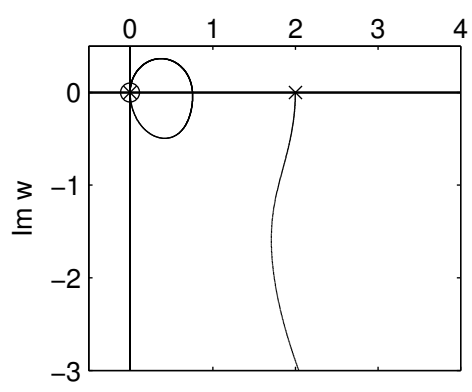

(e) $\varepsilon=1$

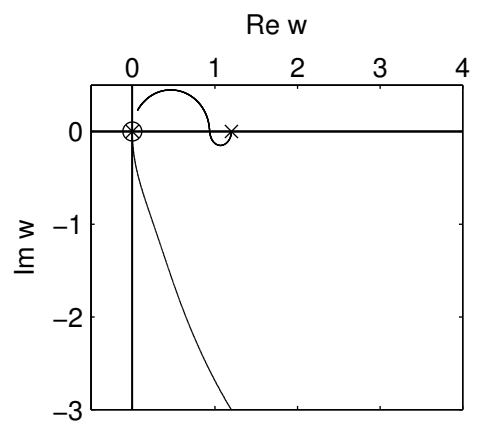

(b) $\varepsilon=0.2$

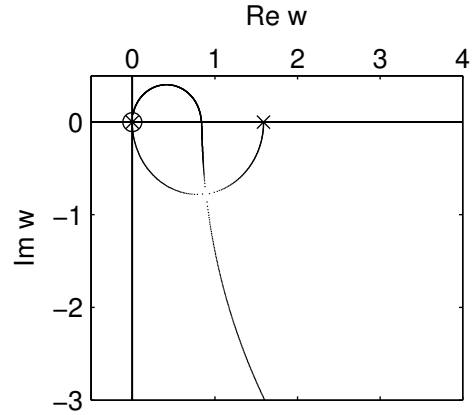

(d) $\varepsilon=0.58$

Re w

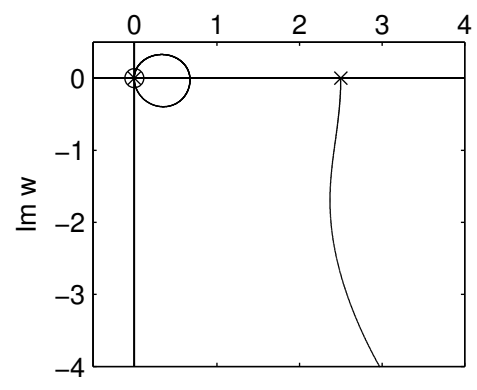

(f) $\varepsilon=1.5$

Figure 3: The longitudinal squared wave speeds of isotropic generalized temperaturerate-dependent thermoelasticity: Model 1. For each part, $\alpha_{0}=0.1, \alpha_{1}=0.2, \tau_{0}=0.1$. 


\section{Generalized temperature-rate-dependent thermoe- lasticity: Model 2}

Following Ignaczak [6] in the isotropic case, we combine the heat-flux constitutive equation (42) of generalized thermoelasticity with the field equations (2) of temperature-ratedependent thermoelasticity to obtain the following field equations for the anisotropic case

$$
\begin{aligned}
& \tilde{c}_{i j k l} u_{k, j l}-\beta_{i j}\left(\theta_{, j}+\alpha_{1}^{\prime} \dot{\theta}_{, j}\right)=\rho \ddot{u}_{i}, \\
& k_{i j} \theta_{, i j}=\left(1+\tau_{1}^{\prime} \frac{\partial}{\partial t}\right)\left(T \beta_{i j} \dot{u}_{i, j}+\rho c\left(1+\alpha_{0}^{\prime} \frac{\partial}{\partial t}\right) \dot{\theta}\right) .
\end{aligned}
$$

The relaxation time $\tau_{1}^{\prime}$ here plays exactly the same role as does $\tau_{0}^{\prime}$ in (42) and (43) and the new notation is selected simply to differentiate between this section and the previous one. If we take $\tau_{1}^{\prime}=0$ then (64) reverts to (2) of temperature-rate-dependent thermoelasticity and if we take $\alpha_{1}^{\prime}=\alpha_{0}^{\prime}=0,(64)$ reverts to the equations of generalized thermoelasticity, see [13].

\subsection{The secular equation}

Similarly to the two previous sections, by inserting (4) into (64) we will get the field equations of isotropic generalized temperature-rate-dependent thermoelasticity: Model 2.

$$
\begin{aligned}
& (\tilde{\lambda}+\tilde{\mu}) u_{j, i j}+\tilde{\mu} u_{i, j j}-\beta\left(\theta+\alpha_{1} \dot{\theta}\right)_{, i}=\rho \ddot{u}_{i}, \\
& k \theta_{, i i}-T \beta\left(\dot{u}_{j, j}+\tau_{1}^{\prime} \ddot{u}_{j, j}\right)-\rho c\left(\dot{\theta}+\left(\alpha_{0}^{\prime}+\tau_{1}^{\prime}\right) \ddot{\theta}+\alpha_{0}^{\prime} \tau_{1}^{\prime} \dddot{\theta}\right)=0 .
\end{aligned}
$$

We now seek solutions of (65) in the form of plane harmonic waves (6) and follow the same steps as in the previous sections to obtain the non-dimensional secular equation

$$
(w-\tilde{\mu})^{2}\left[1-w+\varepsilon \frac{w\left(1-i \omega \alpha_{1}\right)\left(1-i \omega \tau_{1}\right)}{w\left(1-i \omega \alpha_{0}\right)\left(1-i \omega \tau_{1}\right)+i \omega}\right]=0
$$

in which $\tau_{1}=\tau_{1}^{\prime} \omega^{*}$ is another dimensionless relaxation time. The two transverse waves $w=\tilde{\mu}$ are as before. If we take $\tau_{1}=0$ then (66) reverts to (19) of temperature-ratedependent thermoelasticity and if we take $\alpha_{1}^{\prime}=\alpha_{0}^{\prime}=0$, (66) reverts to the equations of generalized thermoelasticity, see [8].

After expanding and rearranging the part within square brackets of equation (66)

we obtain an alternative form of the non-dimensional secular equation for longitudinal waves in isotropic generalized temperature-rate-dependent thermoelasticity: Model 2.

$$
w(w-1-\varepsilon)+i \omega \frac{1+w\left(\alpha_{1}-\alpha_{0}\right)\left(1-i \omega \tau_{1}\right)}{\left(1-i \omega \alpha_{1}\right)\left(1-i \omega \tau_{1}\right)}(w-1)=0 .
$$

This can be rearranged as a quadratic equation in $w$ :

$$
w^{2}\left(1-i \omega \alpha_{0}\right)\left(1-i \omega \tau_{1}\right)-w\left[\left(1-i \omega \alpha_{0}\right)\left(1-i \omega \tau_{1}\right)+\varepsilon\left(1-i \omega \alpha_{1}\right)\left(1-i \omega \tau_{1}\right)-i \omega\right]-i \omega=0
$$

with roots

$$
w_{0,1}=\frac{z_{3} \mp\left[z_{3}^{2}+4 i \omega\left(1-i \omega \alpha_{0}\right)\left(1-i \omega \tau_{1}\right)\right]^{\frac{1}{2}}}{2\left(1-i \omega \alpha_{0}\right)\left(1-i \omega \tau_{1}\right)}
$$

where

$$
z_{3}=\left(1-i \omega \alpha_{0}\right)\left(1-i \omega \tau_{1}\right)+\varepsilon\left(1-i \omega \alpha_{1}\right)\left(1-i \omega \tau_{1}\right)-i \omega
$$


The uncoupled case $\varepsilon=0$. In this case the roots (69) become, from (67),

$$
w_{0}=\frac{-i \omega}{\left(1-i \omega \alpha_{0}\right)\left(1-i \omega \tau_{1}\right)}, \quad w_{1}=1,
$$

the latter corresponding to an unattenuated, non-dispersive longitudinal elastic mode. On decomposing $(70)_{1}$ into its real and imaginary parts $w_{0}=u_{0}+i v_{0}$ we find on eliminating $\omega$ that $w_{0}$ describes the full circle

$$
\left(u_{0}-\frac{1}{2\left(\alpha_{0}+\tau_{1}\right)}\right)^{2}+v_{0}^{2}=\left(\frac{1}{2\left(\alpha_{0}+\tau_{1}\right)}\right)^{2}
$$

in the complex $w$-plane as $\omega$ ranges over positive values, beginning at the origin $(\omega=0)$ and finishing also at the origin $(\omega \rightarrow \infty)$. The real axis is intersected at $u_{0}=1 /\left(\alpha_{0}+\tau_{1}\right)$, $v_{0}=0$, corresponding to the frequency $\omega=1 / \sqrt{\alpha_{0} \tau_{1}}$. This mode is diffusive for small $\omega$ but becomes elastic as $\omega \rightarrow 1 / \sqrt{\alpha_{0} \tau_{1}}$. For $0 \leq \omega \leq 1 / \sqrt{\alpha_{0} \tau_{1}}, w_{0}$ describes a semicircle in the lower half $w$-plane and so represents a stable mode, whereas for $1 / \sqrt{\alpha_{0} \tau_{1}}<\omega<\infty$, $w_{0}$ describes a semicircle in the upper half $w$-plane and so represents an unstable mode. This is illustrated in Figure 4(a).

\subsection{Low frequency expansions}

At zero frequency the roots (69) are given once again by (28) for $\varepsilon>0$, as is most easily seen by putting $\omega=0$ in (67). These roots are marked $\times$ in Figure 4 , in which the roots (69) are plotted in the complex $w$-plane for various values of $\varepsilon \geq 0$.

From (69) for small $\omega$, we find that

$$
w_{0}=\frac{-i \omega}{1+\varepsilon}+O\left(\omega^{2}\right)
$$

and that

$$
w_{1}=1+\varepsilon-i \omega \varepsilon\left\{\alpha_{1}-\alpha_{0}+\frac{1}{1+\varepsilon}\right\}+O\left(\omega^{2}\right) .
$$

These modes are identical to (29) and (30), respectively, in the temperature-rate-dependent case and so both are stable at low frequency. The presence of the additional relaxation time $\tau_{1}$ does not affect the low frequency results at $O(\omega)$.

\subsection{High frequency expansions}

We explore the high frequency domain $\omega \rightarrow \infty$ by putting $\omega=1 / \zeta$ in (68), multiplying by $\zeta^{2}$ to obtain

$$
w^{2}\left(\zeta-i \alpha_{0}\right)\left(\zeta-i \tau_{1}\right)-w\left[\left(\zeta-i \alpha_{0}\right)\left(\zeta-i \tau_{1}\right)+\varepsilon\left(\zeta-i \alpha_{1}\right)\left(\zeta-i \tau_{1}\right)-i \zeta\right]-i \zeta=0,
$$

and then taking the limit $\zeta \rightarrow 0$. Putting $\zeta=0$ in (74) reduces it to

$$
w^{2}-w\left(1+\varepsilon \frac{\alpha_{1}}{\alpha_{0}}\right)=0
$$

with roots

$$
w_{0}=0, \quad w_{1}=1+\varepsilon \frac{\alpha_{1}}{\alpha_{0}}
$$


both of which are marked $\circ$ in each part of Figure 4 . Thus the origin is marked by both $\times$ and $\circ$ in each part of the Figure.

For small $\zeta$ these roots may be better approximated by

$$
w_{0}=i A \zeta+O\left(\zeta^{2}\right), \quad w_{1}=1+\varepsilon \frac{\alpha_{1}}{\alpha_{0}}+i B \zeta+O\left(\zeta^{2}\right)
$$

The constants $A$ and $B$ may be obtained by substitution into (74) to arrive finally at the high frequency approximations

$$
\begin{gathered}
w_{0}=\frac{i \omega^{-1}}{\alpha_{0} \tau_{1}}\left(1+\varepsilon \frac{\alpha_{1}}{\alpha_{0}}\right)^{-1}+O\left(\omega^{-2}\right), \\
w_{1}=1+\varepsilon \frac{\alpha_{1}}{\alpha_{0}}+\frac{i \omega^{-1} \varepsilon \alpha_{1}}{\alpha_{0}^{2} \tau_{1}}\left(1+\varepsilon \frac{\alpha_{1}}{\alpha_{0}}\right)^{-1}\left[1-\tau_{1}\left(1+\varepsilon \frac{\alpha_{1}}{\alpha_{0}}\right)\left(1-\frac{\alpha_{0}}{\alpha_{1}}\right)\right]+O\left(\omega^{-2}\right) .
\end{gathered}
$$

Clearly the branch $w_{0}$ is unstable as is illustrated in each part of Figure 4 . The branch $w_{1}$ is stable provided

$$
\tau_{1}>\left(1+\varepsilon \frac{\alpha_{1}}{\alpha_{0}}\right)^{-1}\left(1-\frac{\alpha_{0}}{\alpha_{1}}\right)^{-1} .
$$

Thus for fixed values of $\alpha_{0}, \alpha_{1}$ and $\varepsilon$ the branch $w_{1}$ is unstable for $\tau_{1}$ small enough. However, for $\tau_{1}=0$ the equations of this section reduce to those of temperature-ratedependent thermoelasticity for which all branches are stable.

The inequality (79) can be rewritten as

$$
1+\varepsilon \frac{\alpha_{1}}{\alpha_{0}}>\frac{1}{\tau_{1}}\left(1-\frac{\alpha_{0}}{\alpha_{1}}\right)^{-1} .
$$

Thus for fixed values of $\alpha_{0}, \alpha_{1}$ and $\tau_{1}$ the branch $w_{1}$ could be unstable for $\varepsilon$ small enough, though if

$$
\tau_{1}>\left(1-\frac{\alpha_{0}}{\alpha_{1}}\right)^{-1}
$$

the branch $w_{1}$ is stable for all $\varepsilon \geq 0$. This is illustrated in Figure 4 where $w_{1}$ is unstable for $\varepsilon<1.5$ and stable for $\varepsilon>1.5$. In Figure $4(\mathrm{~d})$, where $\varepsilon=1.5$, the inequalities (79) and (80) are satisfied as equalities (for $\alpha_{0}=0.1, \alpha_{1}=0.2$, and $\tau_{1}=0.5$ ) and so $B=0$ in $(76)_{2}$, so that higher order terms in $\zeta$ must be considered in order to determine stability or otherwise.

\subsection{Intersection of branches}

Points of intersection of the branches of $w(\omega)$ given by (69) occur when

$$
D \equiv z_{3}^{2}+4 i \omega\left(1-i \omega \alpha_{0}\right)\left(1-i \omega \tau_{1}\right)=0
$$

The vanishing of $\operatorname{Im} D$ gives the following expression for the critical frequency $\omega_{c}$ at which the branches intersect:

$$
\omega_{c}^{2}=\frac{(1+\varepsilon) d_{1}-2}{d_{1} e_{1}^{2}-2 \alpha_{0} \tau_{1}}
$$


where $d_{1}$ and $e_{1}$ are defined by

$$
d_{1}=1+\alpha_{0}+\tau_{1}+\varepsilon\left(\alpha_{1}+\tau_{1}\right), \quad e_{1}=\left(\alpha_{0}+\varepsilon \alpha_{1}\right) \tau_{1} .
$$

The vanishing of $\operatorname{Re} D$ gives

$$
(1+\varepsilon)^{2}-\omega^{2}\left[d_{1}^{2}+2(1+\varepsilon) e_{1}^{2}-4\left(\alpha_{0}+\tau_{1}\right)\right]+\omega^{4} e_{1}^{2}=0,
$$

which on eliminating $\omega$ using (81) gives an equation of eighth degree in $\varepsilon$ (unless $\alpha_{1} \tau_{1}=$ $1 / \sqrt{2}$ ), a positive real root of which yields a value $\varepsilon_{c}$ at which the two branches intersect at the frequency $\omega_{c}$ given by (81). This is illustrated in Figure $4(\mathrm{~d})$ in which we see that for $\alpha_{0}=0.1, \alpha_{1}=0.2$ and $\tau_{1}=0.5$ the branches intersect for $\varepsilon_{c} \approx 0.179$.

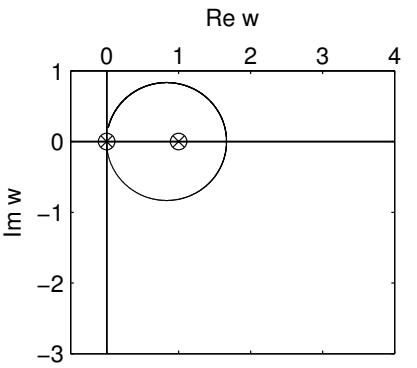

(a) $\varepsilon=0$

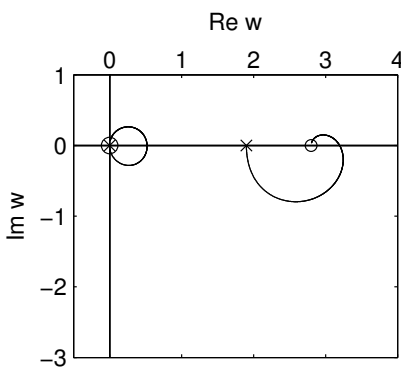

(c) $\varepsilon=0.9$

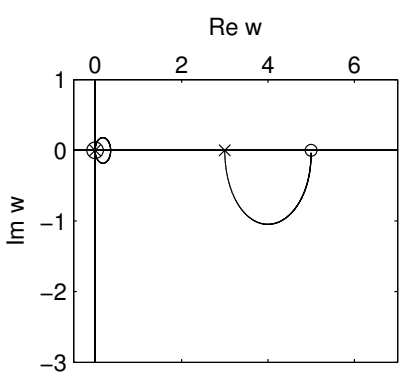

(e) $\varepsilon=2$

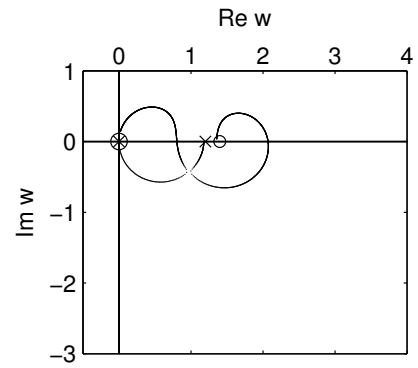

(b) $\varepsilon=0.179$

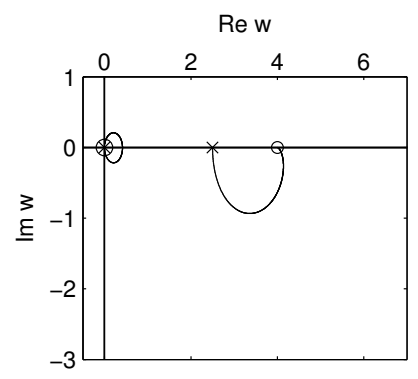

(d) $\varepsilon=1.5$

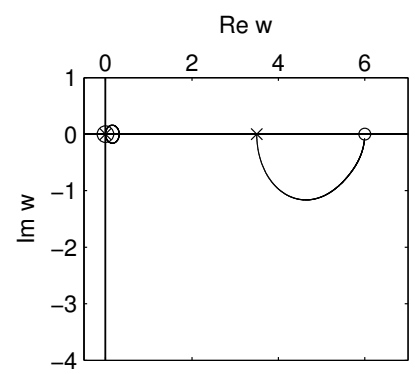

(f) $\varepsilon=2.5$

Figure 4: The longitudinal squared wave speeds of isotropic generalized temperaturerate-dependent thermoelasticity: Model 2. For each part, $\alpha_{0}=0.1, \alpha_{1}=0.2, \tau_{1}=0.5$. 


\section{Discussion}

We saw in Section 2 that Green and Lindsay's [2] theory of temperature-rate-dependent thermoelasticity is linearly stable in the isotropic case, that is, the amplitude of an initially small disturbance remains uniformly bounded in the direction of travel for all time. Leslie and Scott have previously demonstrated the linear stability of isotropic classical thermoelasticity [7, section 2], see also Chadwick [9], so that the present work is an extension of these results. Furthermore, Leslie and Scott have demonstrated the linear stability of isotropic generalized thermoelasticity [8, section 2] in which Fourier's law of heat conduction is replaced by (42), thereby introducing a relaxation time.

In Section 3, however, we saw that the model we proposed for generalized temperaturerate-dependent thermoelasticity on the basis of Chandrasekharaiah and Keshavan's [5] unified system of governing equations is not linearly stable, one of the two longitudinal waves being stable for all frequencies and the other unstable at high frequencies.

In any material we might expect an initial small disturbance either to decay to zero or remain bounded as time increases. Thus we might regard the linear stability of wave forms as being a possible criterion for physically reasonable response. We have seen that both classical and generalized thermoelasticity possess this property and that Green and Lindsay's [2] theory of temperature-rate-dependent thermoelasticity also shares this property. However, in Section 3 we saw that generalized temperature-rate-dependent thermoelasticity: Model 1 does not possess this property. Therefore, we might conclude that this is not a good physical model; this is hardly surprising as it is based on a system of equations (47), see also [5, (2.3)], that has no physical basis.

In Section 4 we considered an alternative theory of generalized temperature-ratedependent thermoelasticity put forward originally by Ignacak [6] in which he combined the field equations of temperature-rate-dependent thermoelasticity (46) with the heat conduction equation (42) of generalized thermoelasticity. We saw that this procedure too led to one or both of the longitudinal waves being unstable at high frequencies. This is somewhat surprising as Ignacak's theory is rationally based and was obtained in exactly the same way that the (stable wave) theory of generalized thermoelasticity, see [8], is obtained from the classical thermoelasticity equations (1) and the heat conduction equation of generalized thermoelasticity (42). We cannot explain this lack of linear stability in a theory that seems otherwise to be rationally based.

Acknowledgement A.M. Alharbi wishes to thank Taif University and the Saudi Arabian Ministry of Higher Education for their financial support during her $\mathrm{PhD}$ studies. The authors would like to thank the referees for their helpful suggestions. 


\section{References}

[1] Lord, HW and Shulman, Y. A generalized dynamical theory of thermoelasticity, J. Mech. Phys. Solids, 15 (1967) 299-309.

[2] Green, AE and Lindsay, KA. Thermoelasticity, J. Elasticity, 2 (1972) 1-7.

[3] Straughan, B. Heat Waves. New York, Dordrecht, Heidelberg, London: Springer (2011).

[4] Chandrasekharaiah, DS. Thermoelasticity with second sound: A review, Applied Mechanics Reviews, 39 (1986) 355-376.

[5] Chandrasekharaiah, DS and Keshavan, HR. Thermoelastic plane waves in a transversely isotropic body, Acta Mechanica, 87 (1991) 11-22.

[6] Ignaczak, J. Linear dynamic thermoelasticity-a survey, Shock Vibration Digest, 13 (1980) 3-8.

[7] Leslie, DJ and Scott, NH. Incompressibility at uniform temperature or entropy in isotropic thermoelasticity, Q. JI Mech. Appl. Math., 51 (1998) 191-211.

[8] Leslie, DJ and Scott, NH. Wave stability for incompressibility at uniform temperature or entropy in generalized isotropic thermoelasticity, Q. JI Mech. Appl. Math., 53 (2000) 1-25.

[9] Chadwick, P. Interchange of modal properties in the propagation of harmonic waves in heat-conducting materials, Bull. Austral Math. Soc. 8 (1973) 75-92.

[10] Chadwick, P. Basic properties of plane harmonic waves in a prestressed heatconducting elastic material J.Thermal Stresses 2 (1979) 193-214.

[11] Scott, NH. Connections between deformation-temperature and deformationentropy constraints and near-constraints in thermoelasticity, Int. J. engng Sci. 34 (1996) 1689-1704.

[12] Scott, NH. A theorem in thermoelasticity and its application to linear stability, Proc. R. Soc. Lond. A, 424 (1989) 143-153.

[13] Scott, NH. The stability of plane waves in generalised thermoelasticity. In Elastic Wave Propagation, Eds M. F. McCarthy and M. A. Hayes, pp 623-628. NorthHolland, Amsterdam (1989). 